\title{
Formation of Hierarchical Hybrid Silica-Polymer Using Quantitative Cryo- Electron Tomography
}

M-A. Moradi, D. Eren, S. Rzadkievics, A. Keizer, M. M. J van Rijt, P. H. H. Bomans, H. Friedrich, N. A. J. M. Sommerdijk and J. P. Patterson

Laboratory of Materials and Interface Chemistry and Center for Multiscale Electron Microscopy, Eindhoven University of Technology, Den Dolech 2, 5600 MB Eindhoven, The Netherlands.

Bottom up self-assembly is an elegant strategy to create hierarchical hybrid materials, which find application in catalysis, separation science, drug-delivery, etc. For the rational design of these materials, it is essential to understand and control the formation pathway over multiple steps in the assembly. In this research, we aim to understand the formation pathway of silica-polymer binary colloids during self-assembly into hybrid materials, and their subsequent colloidal crystallization under controlled drying conditions.

We use cryogenic transmission electron microscopy (cryoTEM) [1] and cryogenic electron tomography (cryoET) to capture the self-assembly of the silica-polymer binary particles under various solution conditions and follow the intermediate states of the concentrating dispersion. A collection of 67 images is taken at 2-degree steps from -66 to +66 degrees with low electron dose from frozen dispersions. The tomographic reconstruction of the three dimensional structure of concentrating and clustering hybrid material (Fig 1) was used to analyze the formation process. By analyzing the position and organization of particles we gained insight into formation pathways which lead to random ordering or colloidal co-crystallization.

We find that, to achieve colloidal crystals, it is essential to avoid clustering during the intermediate stages of drying. We investigate the effect of evaporation rate, dispersion $\mathrm{pH}$ and zeta potential, substrate interaction and the droplet shape change that will affect the final structure of the material. With this information, we can design and predict pathways for the organization of the two components in our system based on the dispersion and drying conditions and aim for specific hierarchical hybrid materials. Examples of ordered and disordered hybrid/porous material are produced in this research. The time-resolved cryoET imaging can add details of the structure evolution to sum up with the information from parallel liquid phase electron microscopy and describe the binary colloidal self-assembly. [2]

\section{References:}

[1] JP Patterson et al, Chem Res 50 (2017), p. 1495.

[2] The authors acknowledge funding from the 4TU.htm. 


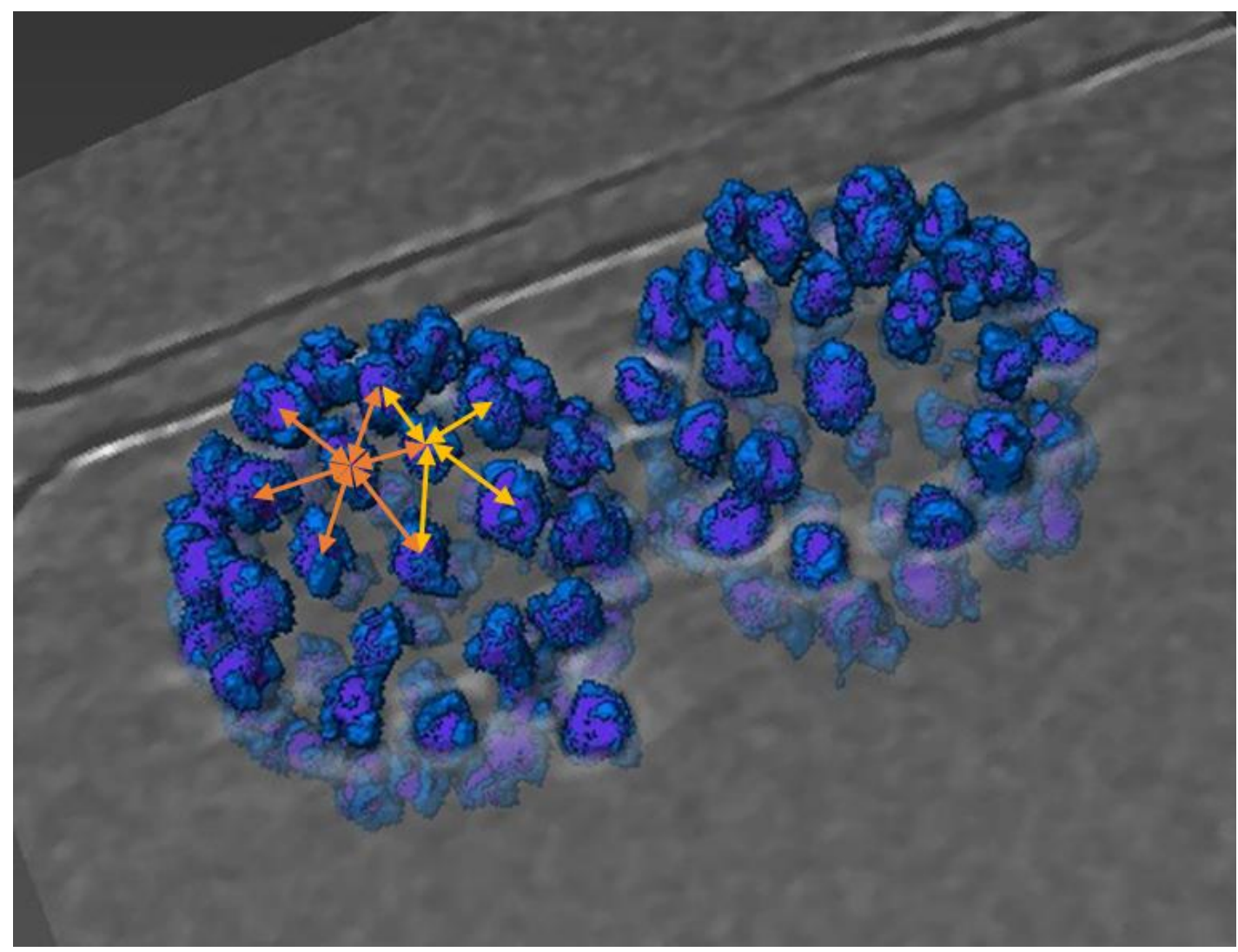

Figure 1. CryoET image of silica-polymer binary particles during controlled drying on a TEM grid. Arrows show a nearest neighbor network of a pentagonal and a hexagonal arrangement of silica particles on the surface of a polymer latex revealed by cryoET. Arrows show an average $30 \mathrm{~nm}$ nearest neighbor distance. 